\title{
RARE EARTH CENTRES IN MIXED COMPOUND CRYSTALS
}

\author{
A. Kozanecki \\ Institute of Physics, Polish Academy of Sciences \\ Al. Lotników 32/46, 02-668 Warsaw, Poland
}

\begin{abstract}
A review of the $\mathrm{Yb}$ impurity related centres in mixed InP-based crystals has been presented. It is shown that $\mathrm{Yb}^{3+}$ ions can be utilized to probe alloy disorder, both in cationic ( $\mathrm{GaInP}$ ) and anionic (InPAs) alloys. The existence of alloy disorder manifested itself in the photoluminescence spectra reflecting local atomic structure of the $\mathrm{Yb}$ centres. The dominant emissions have been ascribed to the $\mathrm{Yb}-\mathrm{P}_{4} \mathrm{Ga}_{12}$ and $\mathrm{Yb}-\mathrm{P}_{4}-\mathrm{Ga}_{11}$ In complexes in $\mathrm{GaInP}$ and to $\mathrm{Yb}-\mathrm{P}_{4}$ and $\mathrm{Yb}-\mathrm{P}_{3}$ As centres in InPAs. The excitation and relaxation mechanisms of the $4 f$-shell of $\mathrm{Yb}^{3+}$ ions were also investigated. It was found that the temperature quenching of the $\mathrm{Yb}^{3+}$ luminescence was accompanied with the appearance of two thermally activated photoluminescence bands. It is suggested that photoluminescence at an energy of $25 \mathrm{meV}$ below the energy gap is due to recombination of excitons bound at Yb-related centres. Relaxation of the excited $4 f$-shell of $\mathrm{Yb}^{3+}$ ions proceeds via back transfer of the excitation energy from the $4 f$-shell to excitons bound at Yb-centres.
\end{abstract}

PACS numbers: 71.55.-i, 78.55.-m, 78.50.Ge

\section{Introduction}

Rare-earth (RE) doped III-V semiconducting compounds are considered as promising materials for potential application in novel light emitting devices, characterized by sharp and temperature stable luminescence originating from the intra- $4 f$-shell emission of $\mathrm{RE}^{3+}$ ions, excited with charge carriers of the semiconductor host. Light emitting diodes operating on the intra- $4 f$-shell electronic transition have been already demonstrated (i.e. $[1,2])$.

The practical use of RE-doped semiconductors requires a high doping level to be achieved. This is due to the long, of the order of milliseconds, radiative lifetime of REs [3]. Doping of covalent semiconductors with REs during crystal growth has met difficulties owing to their high chemical activity. Another problem is associated with the ionic character of bonding of lanthanides to elements of the group V of the atomic periodic table. It results in a structural misfit between the zinc blende type structure of semiconductor hosts and the local structure preferred by RE ions, (usually a cubic, rock salt structure). The structural misfit introduces distortions to 
the lattice and limits seriously the equilibrium solid solubility. Of course, the solid solubility limit can be easily overcome by application of nonequilibrium doping methods, as i.e. ion implantation or doping during MBE and MOCVD growth processes, however, it often results in precipitation of RE-rich metallic phases (i.e. $[4,5])$.

One of the basic problems to be solved is excitation and quenching of the $4 f-4 f$ photoluminescence (PL) of REs in semiconductors. It is believed that excitation of the $4 f$-electron shell of RE ions in semiconductors is accomplished by the transfer of energy from the recombining charge carriers trapped at RE impurities to the $4 f$-electrons $[6,7]$. Therefore, the energy levels in the forbidden gaps associated with lanthanide ions and their electrical character have to be identified. Information indispensable for such identification is the atomic structure of the RE-related centres and, in particular, the location of RE ions in crystalline lattices. In one case only, namely for $\mathrm{Yb}$ in InP, the structure and electrical properties of the RE-related centre seem to be well established. Whitney and co-workers [8] identified an acceptor-like electron trap ( $\mathrm{AE}$ ) due to Yb impurity, located at $30 \mathrm{meV}$ below the conduction band of InP. Aszodi et al. [9] determined the symmetry of the emitting $\mathrm{Yb}^{3+}$ ion as $T_{d}$, suggesting the substitutional position of the $\mathrm{Yb}$ atoms. Kozanecki and Groetzschel [10] confirmed this suggestion using Rutherford backscattering (RBS) and channeling spectroscopy. They also showed that substitutional location of $\mathrm{Yb}$ atoms in InP is an exception for III-V : RE systems. Ytterbium $[10,11]$ and neodymium [12] atoms implanted into gallium compounds (GaP, GaAs, AlGaAs, GaAsP) were found to locate non-substitutionally. Unfortunately, their exact positions could not be determined, because of incomplete annealing of post-implantation damage. It was also found that Er atoms locate close to the tetrahedral interstitial positions in GaAs $[13,14]$, and at the hexagonal interstitial positions in $\mathrm{Si}[5]$.

The spatial extension of the $4 f$-electron wave function in a crystalline host is still of free ion size. This ensures that only the nearest environment of an RE impurity is sampled by its $4 f$-electrons. Because of the screening of the $4 f$-electron states by the outer closed $5 s^{2}$ and $5 p^{6}$ shells, the $4 f$-states are only weakly affected by the crystal field. Nevertheless, the ground and excited states of $\mathrm{RE}^{3+}$ ions are split in a crystal field. As a result, the number of lines and their energy separation reflect the symmetry and intensity of a local electric field. It offers a unique possibility to study perturbations of the highly symmetrical surroundings of the $\mathrm{RE}^{3+}$ ions due to isovalent doping of binary crystals with a third component. The condition necessary to observe the effect of isovalent doping is, apart of the confinement of the electron wave function, the substitutional location of impurity atoms. Therefore, $\mathrm{Yb}$ impurity in InP-based compounds has been selected to study the structure of the RE-related centres in mixed III-V compounds.

An attractive idea is to study excitation and quenching of the intra- $4 f$-shell PL as a function of alloy composition. InPAs alloys are particularly interesting in this respect, as the band gap can be changed between $1.42 \mathrm{eV}(\mathrm{InP})$ and $1.24 \mathrm{eV}$, for the As content of $20 \%$, which is equal to the energy of the $4 f-4 f$ radiative transitions of the $\mathrm{Yb}^{3+}$ ions. 


\section{Ytterbium as a probe of the local alloy disorder in GaInP and InPAs crystals}

It is well known that isovalent impurities added to binary III-V compounds introduce strain that is accommodated differently by the two sublattices [15]. The mixed sublattice tends to form a virtual crystal, while the common sublattice tends to preserve the covalent bond length associated with the nearest-neighbour atoms. As a result, the common sublattice suffers significant bond angle distortions. Because of the different behaviour of the two sublattices as a result of alloying, the reaction of an atomic probe to isovalent doping should depend on its location in one of the sublattices. It is clear at this point that the substitutional position of an impurity atom in both cationic and anionic alloys is a key factor for the possibility to observe the differences in the atomic structure of the centres in both types of alloys.

\subsection{GaInP:Yb alloys}

The effect of Ga-doping on the intra- $4 f$-shell $\mathrm{PL}$ of $\mathrm{Yb}^{3+}$ is presented in Fig. 1 (Ref. [16]). It is seen that the spectra are strongly influenced by the presence of gallium atoms already for $\mathrm{Ga}$ concentrations as low as $0.5 \%$. A comparison of the $\mathrm{Yb}^{3+} \mathrm{PL}$ in $\mathrm{InP}$ (the lowest curve in Fig. 1) and in $\mathrm{Ga}_{x} \operatorname{In}_{1-x} \mathrm{P}(x<10 \%)$, shows that four new lines, denoted with $A-D$, appeared. The intensity of the $4 f-4 f$ PL characteristic of InP decreases considerably as the Ga content increases. The wavelengths and relative intensities of the $A-D$ lines do not seem to depend on composition. Therefore, it is supposed that they are all related to radiative transitions between the sublevels of a single centre.

An ytterbium atom occupying a cation site in GaInP crystals is tetrahedrally coordinated by four $\mathrm{P}$ atoms at the nearest-neighbour (NN) positions and by 12 In and $\mathrm{Ga}$ atoms altogether at the next-nearest-neighbour (NNN) cation sites. Assuming a random statistics of $\mathrm{Ga}$ and In atoms in the mixed sublattice, the probability $P\left(x_{n}\right)$ that $\mathrm{Yb}$ is surrounded by $n$-Ga atoms and $(12-n)$ In atoms is given by the binomial distribution

$$
P\left(x_{n}\right)=\left(\begin{array}{c}
12 \\
n
\end{array}\right) x^{n}(1-x)^{(12-n)},
$$

where $x$ is the composition (gallium content). For $x<10 \%$ the probability of finding an environment with $n \geq 2$ is very low in comparison with that of finding configurations with $n=0$ and $n=1$. A simple analysis of the zinc blende lattice shows that for two Ga atoms in the NNN positions five inequivalent combinations of In and $\mathrm{Ga}$ atoms exist: one for the case when two $\mathrm{Ga}$ atoms are bound to the same $\mathrm{P}$ atom, and four configurations when $\mathrm{Ga}$ atoms are bound to different $P$ atoms. Therefore, it should not be expected that any luminescence transitions related to the presence of two or more $\mathrm{Ga}$ atoms could be resolved in the PL spectrum of $\mathrm{Yb}^{3+}$. As a result, the existence of the configurations with $n \geq 2$ contributes mostly to the broadening of the PL lines [16].

Taking into account these statistical arguments only two independent PL spectra, from $\mathrm{Yb}-\mathrm{P}_{4} \mathrm{Ga}_{12}$ and $\mathrm{Yb}-\mathrm{P}_{4}-\mathrm{Ga}_{11} \mathrm{In}$ centres, can be expected for an 


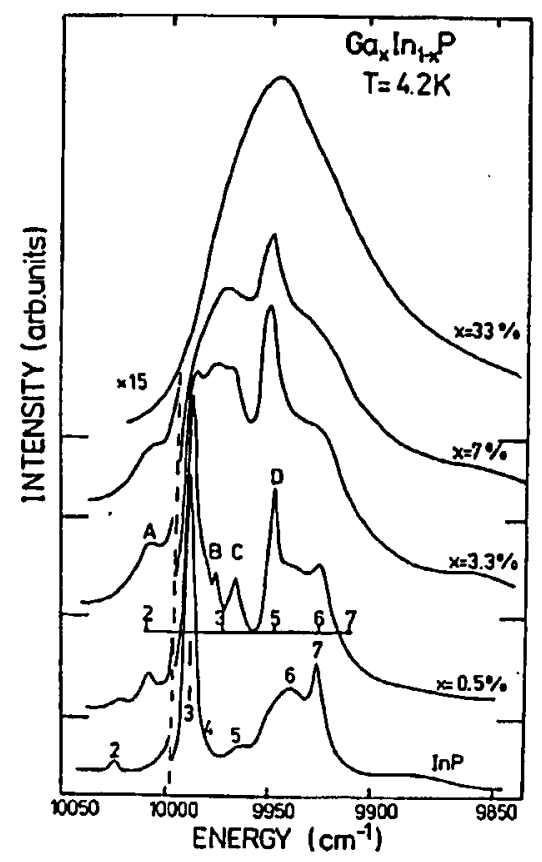

Fig. 1. PL spectra of $\mathrm{Yb}^{3+}$ in $\mathrm{Ga}_{x} \operatorname{In}_{1-x} \mathrm{P}$ for selected compositions.

atomic probe substituting one of the host atoms in a mixed sublattice. This is in accordance with the experimental data presented in Fig. 1 and reported in Ref. [16].

As the bond length in alloys is nearly preserved in the full range of compositions, it can be expected that also the $\mathrm{Yb}-\mathrm{P}$ bond length is nearly constant in $\mathrm{GaInP}$. Therefore, it seems that a replacement of an In atom with a $\mathrm{Ga}$ atom at a site NNN to $\mathrm{Yb}_{\text {In }}$ causes only a local distortion of the $\mathrm{Yb}-\mathrm{P}_{4}$ tetrahedron, as one of the $\mathrm{P}$ atoms $\mathrm{NN}$ to $\mathrm{Y} \mathrm{Yb}^{3+}$ ion has to accommodate three different bond strengths: $\mathrm{P}-\mathrm{Yb}, \mathrm{P}-\mathrm{In}$, and $\mathrm{P}-\mathrm{Ga}$. As a result of this distortion the symmetry of the local electric field is lowered to nearly axial causing splitting of the $\Gamma_{8}$ sublevels in the ground and excited states of $\mathrm{Yb}^{3+}$. Therefore, it is suggested that the $A-D$ $\mathrm{PL}$ lines observed for low Ga admixture (Fig. 1) are due to the $4 f-4 f \mathrm{PL}$ of $\mathrm{Yb}^{3+}$ in axial $\mathrm{Yb}-\mathrm{P}_{4}-\mathrm{Ga}_{11} \mathrm{In}$ complexes [16].

The fact that the volume of the $\mathrm{Yb}-\mathrm{P}_{4}$ tetrahedron does not change remarkably with the crystal composition is confirmed in Fig. 2 in which the $4 f-4 f \mathrm{PL}$ spectra of $\mathrm{Yb}^{3+}$ ions in $\mathrm{InP}, \mathrm{Ga}_{0.5} \mathrm{In}_{0.5} \mathrm{P}$ and $\mathrm{GaP}$ have been presented. It is seen that even for $50 \% \mathrm{Ga}$ content there is no shift of the PL towards higher energies in spite of the decrease in the lattice constant from $5.8687 \AA$ for $\operatorname{InP}$ to $5.6596 \AA$ for $\mathrm{Ga}_{0.5} \mathrm{In}_{0.5} \mathrm{P}$. The $\mathrm{Yb}$ emission in GaP is shifted approximately $20 \mathrm{meV}$ towards higher energies. However, it cannot be decided whether this emission originates from substitutional $\mathrm{Yb}$ atoms, as the dominant sites occupied by $\mathrm{Yb}$ in $\mathrm{GaP}$ are interstitial, according to channeling measurements [11]. 


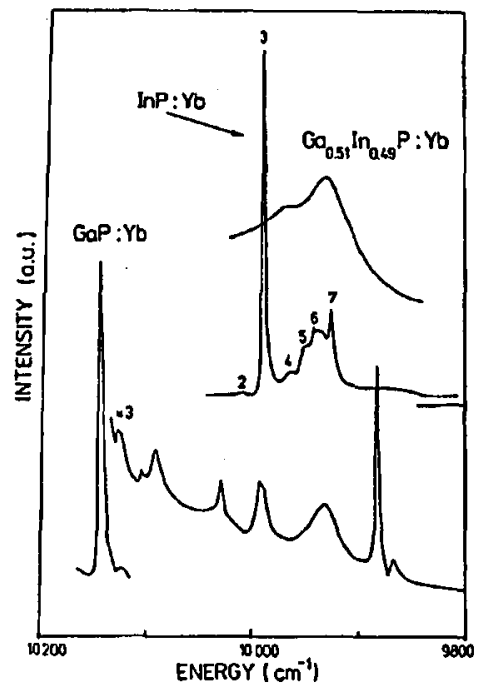

Fig. 2. PL spectra of $\mathrm{Yb}^{3+}$ in $\operatorname{In} \mathrm{P}, \mathrm{GaP}$ and $\mathrm{Ga}_{0.51} \operatorname{In}_{0.49} \mathrm{P}$ crystals.

\subsection{InPAs: $Y b$ alloys}

In the case of InPAs alloy a $\mathrm{P}$-As replacement takes place in a position NN to $\mathrm{Yb}$ atoms. Due to the different bond lengths an As atom replacing one of the $\mathrm{P}$ atoms $\mathrm{NN}$ to an $\mathrm{Yb}^{3+}$ ion induces distortion of the $\mathrm{Yb}$-containing tetrahedron that lowers the symmetry of the cubic $\mathrm{Yb}-\mathrm{P}_{4}$ centre to an axial one. The volume of the distorted $\mathrm{Yb}-\mathrm{P}_{3}$ As tetrahedron should increase slightly with increasing As content to satisfy the expansion of the mixed sublattice according to the Vegard's law. As a result of this expansion a shift of the $4 f-4 f$ emission of $\mathrm{Yb}^{3}+$ towards lower energies can be expected. This suggestion has been confirmed by spectroscopic measurements performed by the author [17-19]. The PL spectra of $\mathrm{Yb}^{3+}$ ions appeared to be extremely sensitive to isovalent doping with arsenic. The addition of arsenic to $\mathrm{InP}$ results in new luminescence transitions (Fig. 3). For the lowest As content (2\%) a weak doublet appeared on the low energy side of the most intense luminescence line at $9987 \mathrm{~cm}^{-1}$, that is ascribed to the $\Gamma_{8}-\Gamma_{8}$ transition of a $\mathrm{Yb}^{3+}$ ion sitting in an undistorted $\mathrm{Yb}-\mathrm{P}_{4}$ tetrahedron. The intensity of this doublet becomes dominant already for a $4 \%$ As admixture. Interestingly, the energy distance between the lines in the doublet is $9 \mathrm{~cm}^{-1}$, and does not seem to depend on crystal composition. In contrast with the behaviour of the new PL in GaInP [16], in InPAs all the new PL lines shift to lower energies with the increase in As contents (Fig. 3). According to the arguments given above this emission is interpreted as originating from axial $\mathrm{Yb}-\mathrm{P}_{3} \mathrm{As}$ complexes [17-19].

An analysis of the temperature dependence of the $\mathrm{Yb}^{3}+\mathrm{PL}$ has given additional proof that the PL spectrum of $\mathrm{Yb}^{3+}$ in InPAs consists of two emissions [17-19], ascribed to two centres: a cubic $\mathrm{Yb}-\mathrm{P}_{4}$ and an axial one $-\mathrm{Yb}-\mathrm{P}_{3} \mathrm{As}$. It was also found that at higher temperatures the intensity of the $\mathrm{PL}$ originating from axial centres decreases in favour of the emission due to the cubic $\mathrm{Yb}-\mathrm{P}_{4}$ complexes. 


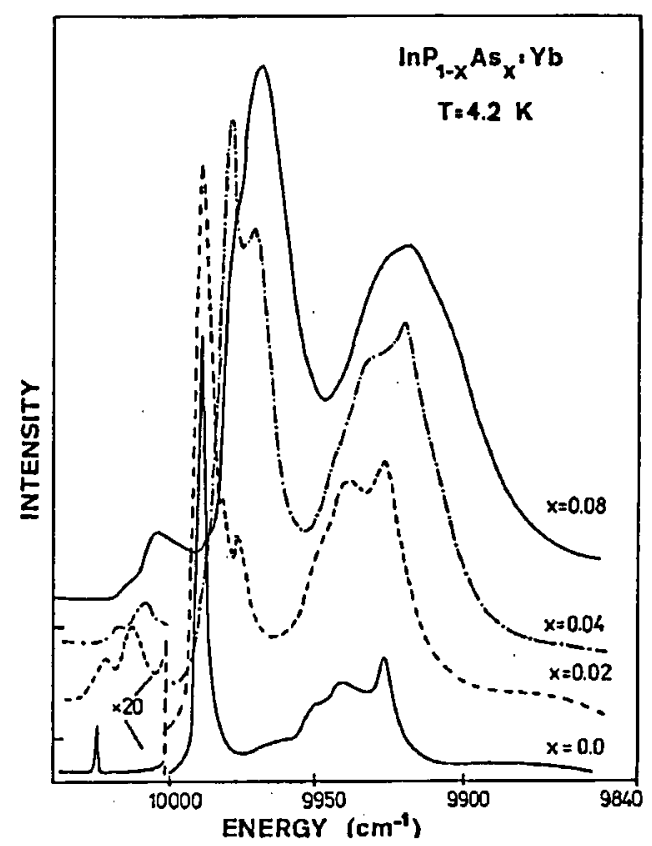

Fig. 3. PL spectra of $\mathrm{Yb}^{3+}$ in selected InPAs crystals.

\section{Excitation and relaxation of the intra- $4 f$-shell $\mathrm{PL}$ of $\mathrm{Yb}^{3+}$ in InP and InPAs alloys}

Yb-doped InP has been most widely used to study the excitation and quenching mechanisms (i.e. $[6,7]$ ) as only one kind of optically active centre due to $\mathrm{Yb}$ impurity has been found and ascribed to $\mathrm{Yb}$ atoms at indium sites [9]. Binding of electrons at $\mathrm{Yb}$ AE-traps has been well documented using optically detected cyclotron resonance [20-22]. Electron paramagnetic resonance experiments revealed a strong $\mathrm{Yb}^{3+}$ signal in both $n$ - and $p$-type $\operatorname{InP}$ [23], indicating that the $30 \mathrm{meV}$ AE-trap cannot be due to the $\mathrm{Yb}^{3+} / \mathrm{Yb}^{2+}$ acceptor level. It provides evidence that the bound electron is located outside the atom core and can be considered as eflective-mass-like. Such negatively charged Yb-centres can attract holes with Coulomb forces, forming excitons.

On the basis of these observations a model was proposed, in which the $4 f$-electron shell is excited via non-radiative Auger-type transfer of energy from the exciton bound at a $\mathrm{Yb}^{3+}$ ion $[6,7]$. Time resolved measurements of Taguchi et al. [24] suggest that the dominant quenching mechanism of the $4 f-4 f$ PL of $\mathrm{Yb}^{3+}$ is the back transfer of energy from the excited ${ }^{2} F_{5 / 2}$ state of the $4 f$-shell to band states of InP. Other authors proposed a model in which the energy of the excited ${ }^{2} F_{5 / 2}$ state of $\mathrm{Yb}^{3+}$ is back transferred to excitons bound at $\mathrm{Yb}^{3+}$ ions [7]. However, the trouble with this model is that a PL originating from radiative recombination of such excitons has not been observed yet. The reason for this is quite simple. In the process of back transfer of energy to excitons bound 
at the AE-trap the energy mismatch $\Delta E \approx 150 \mathrm{meV}$ has to be accommodated by absorption of phonons. This mismatch is many times larger than the binding energy of excitons $(\approx 10 \mathrm{meV})$ and electrons at AE-trap $(\approx 30 \mathrm{meV})$. Therefore, radiative recombination of the Yb-bound excitons is hardly possible due to their immediate thermal dissociation at temperatures at which the back transfer of energy becomes efficient ( $T>100 \mathrm{~K}$ in $\mathrm{InP}[24])$. On the other hand, the conditions favouring thermal stability of the Yb-bound excitons can be met in materials with smaller band gaps and, consequently, with a smaller energy mismatch between the $4 f-4 f$ and excitonic emissions. InPAs: Yb crystals are particularly well suited to search for the $\mathrm{Yb}$ bound exciton, as the energy mismatch $\Delta E$ changes continuously from ca. $150 \mathrm{meV}(\mathrm{InP})$ to $0 \mathrm{meV}$ for $\operatorname{InP}_{0.8} \mathrm{As}_{0.2}$.

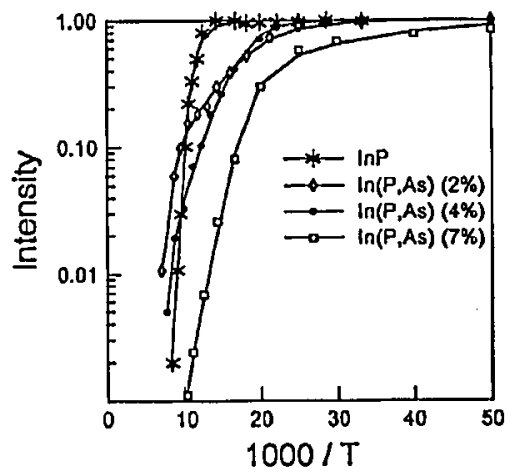

Fig. 4. Semilog- plots of the normalized $\mathrm{Yb}^{3}+\mathrm{PL}$ intensity versus inverse temperature for InPAs.

Temperature dependences of the PL spectra were measured to study the quenching of the $\mathrm{Yb}^{3+} \mathrm{PL}$ in InPAs [25,26]. Figure 4 shows a semilog-plot of the integrated $\mathrm{Yb}^{3+} \mathrm{PL}$ intensity versus inverse temperature for InP and InPAs alloys. Assuming a two-step quenching process of the PL intensity the experimental data were fitted to a relationship

$$
I\left(T_{0}\right) / I(4.2 \mathrm{~K})=\left[1+A_{1} \exp \left(-E_{1} / k T\right)+A_{2} \exp \left(-E_{2} / k T\right)\right]^{-1},
$$

where the prefactors were taken as constant. It has been found that the $E_{1}$ values depend strongly on composition (Table) and are close to the energy mismatch $\Delta E$ between the $4 f-4 f$ transition and the recombination energy of excitons bound at $\mathrm{Yb}^{3+}$ ions. On the other hand, the $E_{2}$ values do not reveal any regular dependence on composition and they are within the range of $10-35 \mathrm{meV}$, which is comparable to the energy position of the AE-trap in the forbidden gap.

In the papers $[18,25,26]$ it was shown that the quenching of the $4 f-4 f \mathrm{PL}$ of $\mathrm{Yb}^{3+}$ in InPAs with $x<8 \%$ is accompanied with the appearance of two thermally stimulated emissions, which were assigned to band-to-band recombination and to recombination of the $e-h$ pairs bound to $\mathrm{Yb}^{3+}$ ions (the emission $E_{\mathrm{Yb}}$ at $25 \mathrm{meV}$ below the band gap $[25,26])$, respectively. This is confirmed by the temperature dependence of $\mathrm{PL}$ in $\mathrm{InP}_{0.88} \mathrm{As}_{0.12}$ (Fig.5). For this composition the $\mathrm{Yb}^{3+} \mathrm{PL}$ 


\section{TABLE}

Fitting parameters for temperature dependence of the $4 f-4 f \mathrm{PL}$ of $\mathrm{Yb}^{3+}$ ions, according to formula (2), and the energy mismatch, $\Delta E$, between the Yb-bound exciton and the $4 f-4 f$ electronic recombination.

\begin{tabular}{l|c|c|c|r|c}
\hline \hline Crystal & $E_{1}[\mathrm{meV}]$ & $A_{1}$ & $E_{2}[\mathrm{meV}]$ & \multicolumn{1}{c|}{$A_{2}$} & $\Delta E[\mathrm{meV}]$ \\
\hline $\mathrm{InP}$ & 190.0 & $3.3 \times 10^{7}$ & 30.0 & 0.1 & 150.0 \\
$\operatorname{InP}_{0.98} \mathrm{As}_{0.02}$ & 138.0 & $1.3 \times 10^{7}$ & 21.0 & 82.0 & 130.0 \\
$\mathrm{InP}_{0.96} \mathrm{As}_{0.04}$ & 81.0 & $7.5 \times 10^{4}$ & 35.0 & 1000.0 & 110.0 \\
$\mathrm{InP}_{0.93} \mathrm{As}_{0.07}$ & 60.0 & $1.1 \times 10^{6}$ & 10.0 & 9.0 & 80.0 \\
$\operatorname{InP}_{0.88} \mathrm{As}_{0.12}$ & 50.0 & & & & 40.0
\end{tabular}
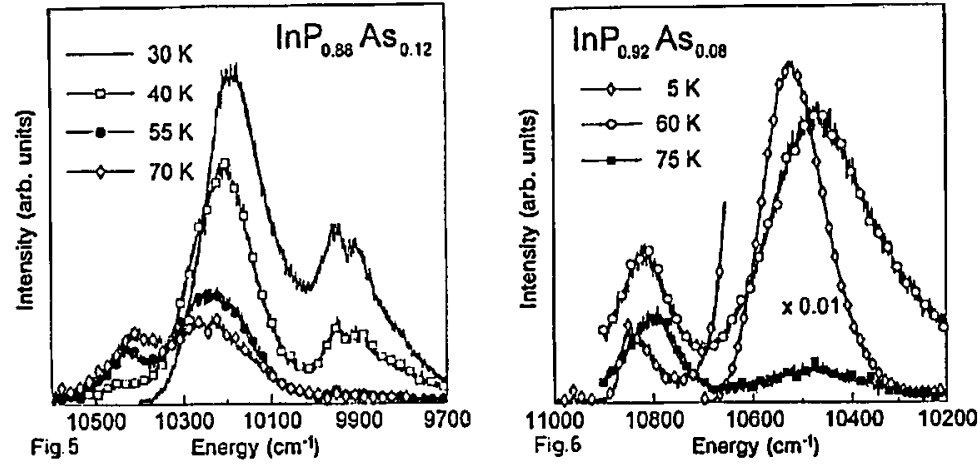

Fig. 5. PL spectra of an $\operatorname{InP}_{0.88} \mathrm{As}_{0.12}$ : Yb crystal.

Fig. 6. Near band-edge luminescence of undoped $\operatorname{InP}_{0.92} \mathrm{As}_{0.08}$ crystal.

disappears already at $55 \mathrm{~K}$, but two emissions in the near band-edge region are observed [27]. To prove directly that the emission at an energy of $25 \mathrm{meV}$ below the band gap is related to $\mathrm{Yb}$, the PL of an $\operatorname{InP}_{0.92} \mathrm{As}_{0.08}$ undoped crystal was measured and the results are presented in Fig. 6. In contrast to the Yb-doped crystal with a similar composition, at $T>50 \mathrm{~K}$ only one thermally stimulated emission was observed in the near band edge region. It is apparently the band-to-band recombination. No PL at an energy of $25 \mathrm{meV}$ less than $E_{\mathrm{g}}$ was detected. Also, no trace of the $\mathrm{Yb}^{3+} \mathrm{PL}$ was observed in the region of $9900-10000 \mathrm{~cm}^{-1}$.

\section{Conclusions}

On the basis of the presented results several conclusions can be drawn:

1. In GaInP alloys the $4 f-4 f$ PL spectra reflect the existence of two emitting $\mathrm{Yb}$-centres: a cubic one $-\mathrm{Yb}-\mathrm{P}_{4} \mathrm{Ga}_{12}$ and an axial one $-\mathrm{Yb}-\mathrm{P}_{4}-\mathrm{Ga}_{11} \mathrm{In}$.

2. In InPAs alloys for each composition the spectrum is interpreted as consisting of two independent emissions due to the $\mathrm{Yb}-\mathrm{P}_{4}$ and $\mathrm{Yb}-\mathrm{P}_{3} \mathrm{As}$ centres.

3. The line splitting $\left(9 \mathrm{~cm}^{-1}\right)$ related to an axial local strain induced by isovalent doping seems to be independent of composition, suggesting that the local strain is alloy composition independent. 
4. It seems that excitation and relaxation of the $4 f$-shell is mediated by an exciton bound to the $\mathrm{Yb}^{3+}$ ion.

5. Near band gap luminescence due to the exciton bound at $\mathrm{Yb}^{3+}$ (or electron trapped at $\mathrm{Yb}^{3+}$ with a free hole) has been identified.

\section{Acknowledgment}

This work has been supported in part by the Committee for Scientific Research (grant No. PB 1027/T11/96/10).

\section{R.eferences}

[1] P.S. Whitney, K. Uwai, H. Nakagome, K. Takahei, Electron. Lett. 24, 740 (1988).

[2] B. Zheng, J. Michel, F.Y.G. Ren, L.C. Kimerling, D.C. Jacobson, J.M. Poate, Appl. Phys. Lett. 64, 2842 (1994).

[3] Y.II. Xie, E.A. Fitzgerald, Y.J. Mii, J. Appl. Phys. 70, 3223 (1991).

[4] A.R. Peaker, II. Eleoglu, J.M. Langer, A.C. Wright, I. Poole, K.E. Singer, in: Rare Earth Doped Semiconductors, Eds. G.S. Pomrenke, P.B. Klein, D.W. Langer, in series Materials Research Society Symposium Proceedings, Vol. 301, MRS, Pittsburgh 1994, p. 337.

[5] A. Kozanecki, R.J. Wilson, B.J. Sealy, J. Kaczanowski, L. Nowicki, Appl. Phys. Lett. 67, 1847 (1995).

[6] K. Takahei, A. Taguchi, H. Nakagome, K. Uwai, P.S. Whitney, J. Appl. Phys. 66, 4941 (1989).

[7] K. Thonke, K. Pressel, G. Bohnert, A. Stapor, J. Weber, A. Mollasioti, A. Hangleiter, F. Scholz, Semicond. Sci. Technol. 5, 1124 (1990).

[8] P.S. Whitney, K. Uwai, H. Nakagome, K. Takahei, Appl. Phys. Lett. 62, 84 (1988).

[9] G. Aszodi, J. Weber, Ch. Uhleilın, L. Pu-lin, H. Ennen, U. Kaufmann, J. Schneider, J. Windscheiff, Pliys. Rev. B 31, 7767 (1985).

[10] A. Kozanecki, R. Groetzschel, J. Appl. Phys. 68, 517 (1990).

[11] A. Kozanecki, R. Groetzschel, J. Appl. Phys. 64, 3315 (1988).

[12] A. Kozanecki, R. Groetzschel, J. Appl. Phys. 69, 1300 (1991).

[13] A. Kozanecki, C. Jeynes, M. Chan, B.J. Sealy, Solid State Commun. 78, 763 (1991).

[14] Y. Kido, T. Islida, E. Nakai, M. Saeki, J. Nakata, K. Takahei, Nucl. Instrum. Methods in Phys. Res. B 85, 484 (1994).

[15] J.C. Mikkelsen, Jr., J.B. Boyce, Phys. Rev. B 28, 7130 (1983).

[16] A. Kozanecki, Z. Kaliński, J. Raczyniska, J.M. Langer, J. Appl. Phys. 66, 3202 (1989).

[17] A. Kozanecki, Z. Kaliniski, in: Proc. 20th Intern. Conf. on the Physics of Semiconductors, Thessaloniki (Greece), 1990, Eds. E.M. Anastassakis, J.D. Joannopoulos, World Scientific, Singapore 1990, p. 1989.

[18] A. Kozanecki, Z. Kaliniski, in: Defects in Semiconductors 17, Mater. Sci. Forum 143-147, Eds. H. Heinrich, W. Jantsch, Trans. Tech. Publ., Aedermansdorf (Switzerland) 1994, p. 737.

[19] A. Kozanecki, in: Rare Earlh Doped Semiconductors, Eds. G.S. Pomrenke, P.B. Klein, D.W. Langer, in series Materials Research Society Symposium Proceedings, Vol. 301, MRS, Pittsburgh 1994, p. 219. 
[20] B.J. Heijmink-Liesert, M. Godlewski, A. Stappor, T. Gregorkiewicz, C.A.J. Ammerlaan, J. Weber, M. Moser, F. Scholz, Appl. Phys. Lett. 58, 2237 (1991).

[21] I. Tsimperidis, T. Gregorkiewicz, C.A.J. Ammerlaan, M. Godlewski, F. Scholz, B. Lambert, J. Appl. Phys. Lett. 77, 1523 (1995).

[22] M. Godlewski, A. Kozanecki, J.P. Bergman, B. Monemar, Appl. Phys. Lett. 66, 493 (1995).

[23] B. Lambert, Y. Toudic, G. Grandpierre, A. Rupert, A. Le Corre, Electron. Lett. 24, 1447 (1988).

[24] A. Taguchi, M. Taniguchi, K. Takahei, Appl. Phys. Lett. 60, 965 (1991).

[25] A. Kozanecki, K. Karpiniska, Z. Kaliński, Appl. Phys. Lett. 62, 84 (1993).

[26] A. Kozanecki, A. Szczerbakow, B. Koziarska, in: Defects in Semiconductors 18, Eds. M. Suezawa, H. Katayama-Yoshida, Mater. Sci. Forum 196-201, 651 (1996).

[27] A. Kozanecki, A. Szczerbakow, Appl. Phys. Lett. c6, 3630 (1995). 